\title{
Number of Metabolic Syndrome Risk Factors is Related to Carotid Intima-Media Thickness in Rheumatoid Arthritis Patients
}

\author{
Ferhat GÖKMEN, ${ }^{1}$ Ahmet TEMİZ, ${ }^{2}$ Ayla AKBAL, ${ }^{1}$ Hacer ŞEN, ${ }^{3}$ Coşkun ZATERİ, ${ }^{1}$ Esra GÖKMEN, ${ }^{4}$ \\ Emre BOZKURT, ${ }^{1}$ Hatice REŞORLU, ${ }^{1}$ Tolga KURT, ${ }^{5}$ Yllmaz SAVAŞ ${ }^{1}$ \\ ${ }^{1}$ Department of Physical Medicine and Rehabilitation, Medical Faculty of Çanakkale Onsekiz Mart University, Çanakkale, Turkey \\ ${ }^{2}$ Department of Cardiology, Medical Faculty of Çanakkale Onsekiz Mart University, Çanakkale, Turkey \\ ${ }^{3}$ Department of Internal Medicine, Medical Faculty of Çanakkale Onsekiz Mart University, Çanakkale, Turkey \\ ${ }^{4}$ Department of Internal Medicine, Çanakkale State Hospital, Çanakkale, Turkey \\ ${ }^{5}$ Department of Cardiovascular Surgery, Medical Faculty of Çanakkale Onsekiz Mart University, Çanakkale, Turkey
}

\begin{abstract}
Objectives: This study aims to investigate the relationship between carotid intima-media thickness (CIMT) and clinical and metabolic variables in rheumatoid arthritis (RA) patients.

Patients and methods: The study included 76 RA patients (18 males, 58 females; mean age $50.1 \pm 9.8$ years; range 21 to 69 years) and 42 control subjects (11 males, 31 females; mean age 49.2 \pm 9.7 ; range 28 to 66 years). Erythrocyte sedimentation rate, C-reactive protein, and disease activity score were used to assess disease activation. Rheumatoid factor, anti-cyclic citrullinated peptide antibodies, and metabolic syndrome components (fasting blood glucose, high density lipoprotein-cholesterol, triglyceride, blood pressure, and waist circumference) were measured.

Results: Mean disease duration was $6.9 \pm 6.5$ years. Patients with RA had higher CIMT than the controls $(0.8 \pm 0.1$ and $0.6 \pm 0.2$, respectively; $p<0.001)$. Statistically significant positive correlations were observed between CIMT and age, erythrocyte sedimentation rate, C-reactive protein, and systolic and diastolic blood pressure. The CIMT in RA patients having metabolic syndrome risk components ranging from one to four were $0.76 \pm 0.16$, $0.82 \pm 0.14,0.86 \pm 0.13$, and $0.92 \pm 0.13$, respectively. CIMT was positively correlated with the number of metabolic syndrome risk components.

Conclusion: Our study has shown elevated CIMT in RA. Presence of metabolic syndrome components in RA patients increases tendency to atherosclerosis and constitutes a severe risk for cardiovascular disease.

Keywords: Cardiovascular risk; carotid intima media thickness; metabolic syndrome risk factors; rheumatoid arthritis.
\end{abstract}

Rheumatoid arthritis (RA) is a systemic inflammatory disease which causes increased risk of cardiovascular disease (CVD), and is characterized by chronic erosive synovitis of the peripheral joints. Important factors in the increased risk of CVD in RA patients are chronic inflammation, drugs used in treatment of RA, and a sedentary lifestyle. ${ }^{1}$ Chronic inflammation is the most important reason for accelerated atherosclerosis development in RA. ${ }^{2}$ Cytokines involved in the systemic circulation in RA have altered function in distant organs such as adipose tissue, liver, and skeletal muscle. Thus, they cause a series of atherogenic changes such as insulin resistance, dyslipidemia, oxidative stress, thrombotic effects, endothelial dysfunction, and injury. Even during remission periods of the disease, systemic cytokine levels were found to be higher than those of normal individuals and they caused continued vascular disease. ${ }^{3}$

In various studies, increased carotid intimamedia thickness (CIMT) has been shown to be a good indicator of atherosclerosis. CIMT has been reported to correlate with myocardial infarction, stroke, and peripheral artery disease. ${ }^{4-7}$ Not only is increased CIMT indicative of early atherosclerosis, 
but it also reflects the compensatory enlargement of non-atherosclerotic tissue with thickened medial fibrocellular hypertrophy related to smooth muscle cell hyperplasia and hypertrophy. ${ }^{4,8}$ Increased CIMT and the presence of focal plaque in patients with RA not having traditional CVD risk factors may be a harbinger of cardiovascular events. ${ }^{9,10}$ In the conducted studies, a strong correlation has been shown between the presence of subclinical atherosclerosis and the duration and severity of inflammation and the level of acute phase reactants. ${ }^{1}$

Metabolic syndrome (MetS) is a disorder characterized by a co-occurrence of three out of five of the following medical conditions: abdominal obesity, elevated blood pressure, high serum triglycerides, low high-density cholesterol levels, and elevated fasting plasma glucose. ${ }^{11}$ Some studies showed that increased numbers of MetS risk components lead to worsening cardiovascular disease outcomes and risk factors. ${ }^{12}$ Although CIMT is related to metabolic parameters, only a few studies have investigated the relationship between numbers of MetS risk parameters and CIMT. ${ }^{12}$ Therefore, in this study, we aimed to investigate the relationship between CIMT and clinical and metabolic variables in RA patients.

\section{PATIENTS AND METHODS}

This study was carried out at the Canakkale Onsekiz Mart University Medical Faculty Hospital between April 2012 and January 2013. A total of 118 subjects including 76 RA patients (18 males, 58 females; mean age $50.1 \pm 9.8$ years; range 21 to 69 years) and 42 healthy individuals (11 males,

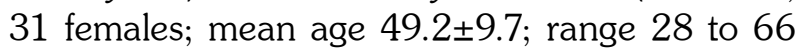
years) were enrolled. All patients gave informed consent forms and the study was approved by the local ethical committee.

Patients were asked about their age, sex, height, weight, body mass index, waist circumference, systolic and diastolic blood pressure, duration of illness, smoking and alcohol consumption status, presence of accompanying chronic diseases, morning stiffness in the joints and duration, and the drugs they continued to use. Laboratory and clinical parameters such as erythrocyte sedimentation rate (ESR), C-reactive protein (CRP), Health Assessment Questionnaire score, and Disease activity score 28 were used in assessing disease activation. Pain evaluation was done using a visual analog scale with a range from 0 to $100 \mathrm{~mm}$. Rheumatoid factor and anti-cyclic citrullinated peptide were measured. MetS was defined according to the National Education Program/Adult Treatment Panel III criteria and MetS components [glucose higher than $110 \mathrm{mg} / \mathrm{dL}$, high-density cholesterol less than $40 \mathrm{mg} / \mathrm{dL}$ (males) or less than $50 \mathrm{mg} / \mathrm{dL}$ (females), triglycerides higher than $150 \mathrm{mg} / \mathrm{dL}$, blood pressure higher than $130 / 80 \mathrm{mmHg}$ or medication for hypertension and waist circumference of $88 \mathrm{~cm}$ for females or $102 \mathrm{~cm}$ for males] were measured. ${ }^{11}$ Exclusion criteria consisted of age $\leq 18$ years, chronic liver/kidney disease, central nervous system disease, malignancy, patients with other inflammatory diseases, and patients who refused to participate in the study.

Echocardiographic measurements with a linear-array imaging probe (GE-vingmed Vivid 7, GE Vingmend Ultrasound AS, Horten, Norway) were employed to evaluate the CIMT of the right common carotid artery. A region $10 \mathrm{~mm}$ proximal to the carotid bifurcation was identified, and the CIMT of the far wall was calculated as the distance between the lumen-intima and media-adventitia interfaces. A frozen image distinctly indicating the borders of the intima and media was obtained and magnified to maximize resolution. CIMT was measured on this image at three adjacent sites $1 \mathrm{~mm}$ apart, and the mean value of the three measurements was used for analyses. All measurements were performed by the same investigator, who was blinded to all patient data.

\section{Statistical analysis}

Analysis of data was performed with SPSS version 15.0 software program (SPSS Inc., Chicago, IL, USA). Normal distribution of data was examined using the KolmogorovSmirnov and Shapiro-Wilk tests. Descriptive variables were expressed as mean, standard deviation, median, minimum, maximum, frequency, and percentage values. Variables normally distributed between groups were analyzed using the Student's t test and nonnormally distributed variables using the MannWhitney $\mathrm{U}$ test. The Chi-square test was used for categorical variables. Correlation between 
Table 1. Comparison of demographic and clinical parameters of groups

\begin{tabular}{|c|c|c|c|c|c|c|}
\hline \multirow[t]{2}{*}{ Variables } & \multicolumn{3}{|c|}{ Patient $(\mathrm{n}=76)$} & \multicolumn{2}{|c|}{ Control $(n=42)$} & \multirow[b]{2}{*}{$p$} \\
\hline & $\mathrm{n}$ & $\%$ & Mean \pm SD & $\%$ & Mean \pm SD & \\
\hline Age (years) & & & $50.1 \pm 9.8$ & & $49.2 \pm 9.7$ & 0.642 \\
\hline Female & & 76.3 & - & 73.8 & - & 0.762 \\
\hline Body mass index $\left(\mathrm{kg} / \mathrm{m}^{2}\right)$ & & & $29.2 \pm 5.4$ & & $28.3 \pm 5.4$ & 0.378 \\
\hline Waist circumference $(\mathrm{cm})$ & & & $95.2 \pm 9.9$ & & $93.7 \pm 6.6$ & 0.379 \\
\hline Systolic blood pressure $(\mathrm{mmHg})$ & & & $131.3 \pm 24.8$ & & $120.4 \pm 16.8$ & 0.013 \\
\hline Diastolic blood pressure $(\mathrm{mmHg})$ & & & $79.4 \pm 13.0$ & & $75.8 \pm 8.5$ & 0.106 \\
\hline Fasting blood glucose (mg/dL) & & & $91.1 \pm 18.4$ & & $96.2 \pm 12.9$ & 0.087 \\
\hline Triglyceride $(\mathrm{mg} / \mathrm{dL})$ & & & $108.8 \pm 50.1$ & & $117.7 \pm 62.7$ & 0.405 \\
\hline High-density lipoprotein cholesterol $(\mathrm{mg} / \mathrm{dL})$ & & & $58.6 \pm 17.6$ & & $57.4 \pm 14.1$ & 0.698 \\
\hline Carotid intima-media thickness (mm) & & & $0.81 \pm 0.14$ & & $0.61 \pm 0.15$ & $<0.001$ \\
\hline Erythrocyte sedimentation rate ( $\mathrm{mm} /$ hour) & & & $39.2 \pm 20.1$ & & $22.7 \pm 17.2$ & $<0.001$ \\
\hline C-reactive protein $(\mathrm{mg} / \mathrm{dL})$ & & & $2.0 \pm 2.4$ & & $0.6 \pm 0.5$ & $<0.001$ \\
\hline Rheumatoid factor positivity & 55 & 72.4 & - & & - & - \\
\hline Anti-CCP positivity & 53 & 69.7 & - & & - & - \\
\hline Disease activity score 28 & & & $4.0 \pm 1.3$ & & - & - \\
\hline Health assessment questionnaire & & & $0.71 \pm 0.61$ & & - & - \\
\hline Visual analog scale $(\mathrm{mm})$ & & & $36 \pm 24$ & & - & - \\
\hline
\end{tabular}

variables in the patient group was analyzed using Spearman's correlation test. $\mathrm{P}<0.05$ was regarded as statistically significant.

\section{RESULTS}

Mean disease duration of RA patients was $6.9 \pm 6.5$ years. Demographic and clinical parameters of the subjects are given in Table 1 . Patients with RA had higher systolic blood pressure, CIMT (Figure 1), ESR, and CRP values than those of the control subjects $(p=0.013, p<0.001, p<0.001$, $p<0.001$, respectively).

A correlation analysis of the relationship between CIMT and clinical parameters (Table 2) revealed significant correlations between CIMT and age $(r=0.381, p<0.001)$, ESR $(r=0.297, p=001)$, CRP $(r=0.429, \quad p<0.001)$, systolic $(r=0.353$, $p<0.001)$ and diastolic blood pressure $(r=0.294$, $\mathrm{p}=0.001)$. MetS frequency was detected in $25 \mathrm{RA}$ patients (32.9\%) and 11 control subjects (26.2\%) $(p=0.449)$. We evaluated the relationship between the number of MetS components and CIMT only in RA patients. We observed that CIMT increased when the number of MetS components increased. The CIMT in RA patients having MetS components ranging from one to four were $0.76 \pm 0.16,0.82 \pm 0.14,0.86 \pm 0.13$, and $0.92 \pm 0.13$, respectively $(p=0.13)$. We demonstrated that CIMT was positively correlated with the number of MetS components ( $p=0.018, r=0.271)$. CIMT according to the number of MetS components in patients with RA is shown in Figure 2.

\section{DISCUSSION}

In the present study, we detected higher CIMT in patients with RA. Additionally, there was a

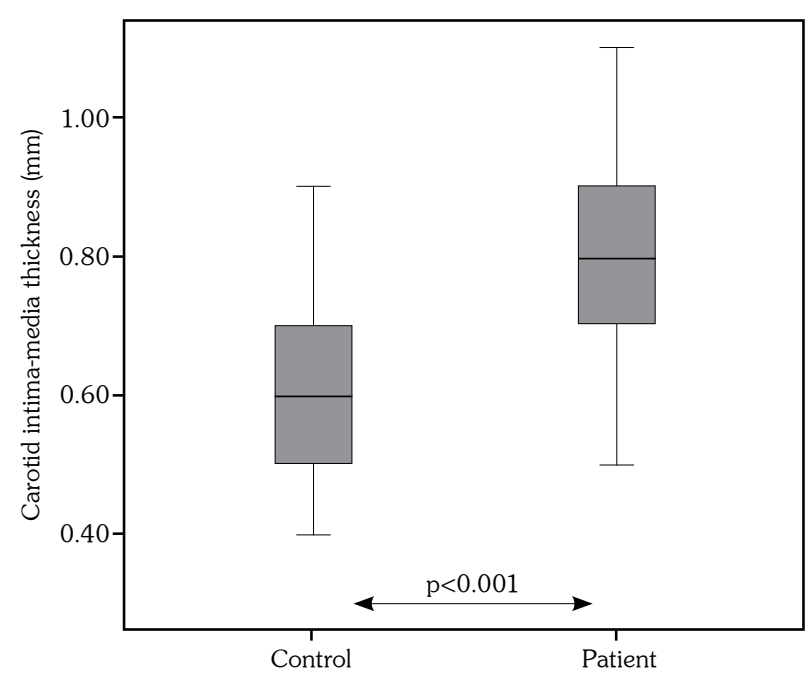

Figure 1. Carotid intima-media thickness values in rheumatoid arthritis and control groups. 


\begin{tabular}{lrr} 
Table 2. Correlations between carotid intima-media \\
thickness and clinical and laboratory values \\
\hline & \multicolumn{2}{c}{ CIMT } \\
\cline { 2 - 3 } & \multicolumn{1}{c}{ r } & $p$ \\
\hline Age (year) & 0.381 & $<0.001$ \\
Waist circumference (cm) & 0.168 & 0.069 \\
Systolic blood pressure (mmHg) & 0.353 & $<0.001$ \\
Diastolic blood pressure (mmHg) & 0.294 & 0.001 \\
Fasting blood glucose (mg/dL) & -0.015 & 0.871 \\
Triglyceride (mg/dL) & 0.041 & 0.658 \\
High-density lipoprotein cholesterol (mg/dL) & -0.087 & 0.354 \\
Erythrocyte sedimentation rate (mm/hour) & 0.297 & 0.001 \\
C-reactive protein (mg/dL) & 0.429 & $<0.001$ \\
Disease activity score 28 & 0.078 & 0.501 \\
Health assessment questionnaire & 0.077 & 0.509 \\
\hline CIMT: Carotid intima-media thickness. & \multicolumn{3}{l}{} \\
\hline
\end{tabular}

positive correlation between CIMT and both ESR and CRP. The study also showed that CIMT gradually increased with the number of MetS components from one to four and there was a positive correlation between CIMT and risk number for MetS.

The incidence of CVD in RA patients is independent of traditional cardiovascular risk factors. ${ }^{13}$ Cardiovascular mortality has been correlated with greater activity of the disease. The inflammation that occurs in persons with RA has been suggested to accelerate the atherosclerotic process. Measurement of CIMT is a useful noninvasive marker of atherosclerotic disease. Previous studies demonstrated that increased common CIMT is a good indicator of generalized atherosclerosis and coronary artery disease. ${ }^{14}$ CIMT has been related to several cardiovascular risk factors, including obesity, hyperlipidemia, and type 2 diabetes mellitus and insulin resistance. Studies have previously reported the presence of subclinical atherosclerosis, confirmed by increased CIMT, in RA patients without a clinical history of cardiovascular events. ${ }^{15,16}$ In the study of Del Rincón et al., ${ }^{17}$ authors showed increased CIMT in patients with RA and a relationship with disease duration. We have shown that disease duration is not related to CIMT. This result may be explained by the short duration of disease. Previous studies showed not only disease duration to be an important risk factor, but also inflammation severity, CRP level, and number of joints involved as significant factors for elevated CIMT. Therefore, we might not have found a

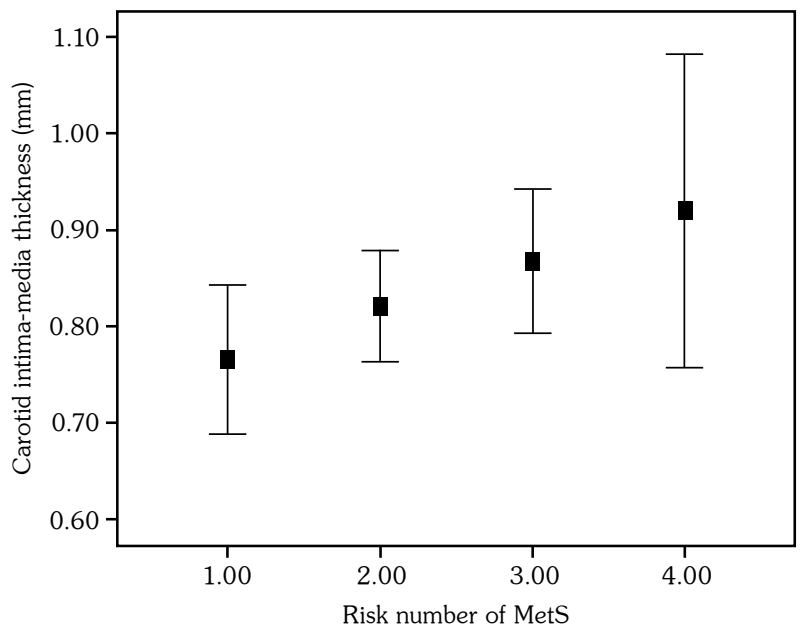

Figure 2. Carotid intima-media thickness values according to number of MetS components in patients with rheumatoid arthritis.

link between CIMT and disease duration due to other factors. However, in our study, CIMT was higher in RA patients than in the control group, demonstrating the presence of subclinical atherosclerosis in RA patients, and corroborating the findings of a majority of the previous work in this field.

The association between carotid atherosclerosis and inflammatory markers such as ESR and CRP has been shown to be independent of cardiovascular risk factors. ${ }^{18}$ A strong correlation was identified between the presence of subclinical atherosclerosis and the magnitude and duration of the inflammatory response obtained by evaluating CRP levels in RA patients. ${ }^{19}$ In our study, we have detected a weak correlation between CIMT and ESR and CRP as clinical parameters indicating inflammation. This is a significant result regarding the inflammatory response correlating with the atherosclerotic process and cardiovascular mortality.

The relationship between CIMT and metabolic parameters in RA patients has been widely investigated. Also, a close relationship between CIMT and a number of cardiovascular risk factors has been demonstrated. ${ }^{20}$ The presence of metabolic parameters such as hypertension, diabetes, and hyperlipidemia in patients with RA causes an increased risk of CVD, and markers of disease severity together with metabolic 
parameters are stated to contribute to predicting the risk of developing CVD. ${ }^{21}$ In another study, a significant relationship was found between CIMT and hypertension and abdominal circumference. ${ }^{22}$ In our study, we showed a correlation between CIMT and systolic and diastolic blood pressure from among metabolic parameters in RA patients. These results, when evaluated together with those of other studies, indicate that these metabolic parameters in RA patients are associated with increased CIMT, giving an increased risk of cardiovascular morbidity and mortality. Another important finding from our study was that CIMT was correlated with the number of MetS parameters in RA patients. We detected that CIMT gradually increased with the number of MetS parameters increasing from one to four. Therefore, we hypothesize that CIMT may be related to cardiovascular mortality.

In conclusion, our study has shown elevated CIMT values in RA. We also demonstrated that CIMT correlated with hypertension from among other metabolic parameters. The results of this study suggest that the number of components of MetS present in patients with RA increases the tendency to atherosclerosis, thus constituting a risk for CVD.

\section{Declaration of conflicting interests}

The authors declared no conflicts of interest with respect to the authorship and/or publication of this article.

\section{Funding}

The authors received no financial support for the research and/or authorship of this article.

\section{REFERENCES}

1. del Rincón ID, Williams K, Stern MP, Freeman GL, Escalante A. High incidence of cardiovascular events in a rheumatoid arthritis cohort not explained by traditional cardiac risk factors. Arthritis Rheum 2001;44:2737-45.

2. Stamatelopoulos KS, Kitas GD, Papamichael CM, Chryssohoou E, Kyrkou K, Georgiopoulos G, et al. Atherosclerosis in rheumatoid arthritis versus diabetes: a comparative study. Arterioscler Thromb Vasc Biol 2009;29:1702-8.

3. Turesson C, Jacobsson L, Bergström U. Extra-articular rheumatoid arthritis: prevalence and mortality. Rheumatology (Oxford) 1999;38:668-74.
4. Kuller L, Borhani N, Furberg C, Gardin J, Manolio $\mathrm{T}$, O'Leary $\mathrm{D}$, et al. Prevalence of subclinical atherosclerosis and cardiovascular disease and association with risk factors in the Cardiovascular Health Study. Am J Epidemiol 1994;139:1164-79.

5. Touboul PJ, Elbaz A, Koller C, Lucas C, Adraï V, Chédru F, et al. Common carotid artery intimamedia thickness and brain infarction : the Etude du Profil Génétique de l'Infarctus Cérébral (GENIC) casecontrol study. The GENIC Investigators. Circulation 2000;102:313-8.

6. O'Leary DH, Polak JF, Kronmal RA, Manolio TA, Burke GL, Wolfson SK Jr. Carotid-artery intima and media thickness as a risk factor for myocardial infarction and stroke in older adults. Cardiovascular Health Study Collaborative Research Group. N Engl J Med 1999;340:14-22.

7. Poredos P. Intima-media thickness: indicator of cardiovascular risk and measure of the extent of atherosclerosis. Vasc Med 2004;9:46-54.

8. Homma S, Hirose $\mathrm{N}$, Ishida $\mathrm{H}$, Ishii $\mathrm{T}$, Araki G. Carotid plaque and intima-media thickness assessed by b-mode ultrasonography in subjects ranging from young adults to centenarians. Stroke 2001;32:830-5.

9. Gonzalez-Juanatey C, Llorca J, Martin J, GonzalezGay MA. Carotid intima-media thickness predicts the development of cardiovascular events in patients with rheumatoid arthritis. Semin Arthritis Rheum 2009;38:366-71.

10. Evans MR, Escalante A, Battafarano DF, Freeman GL, O'Leary DH, del Rincón I. Carotid atherosclerosis predicts incident acute coronary syndromes in rheumatoid arthritis. Arthritis Rheum 2011;63:121120.

11. Gonzalez-Gay MA, Gonzalez-Juanatey C, Piñeiro A, Garcia-Porrua C, Testa A, Llorca J. High-grade C-reactive protein elevation correlates with accelerated atherogenesis in patients with rheumatoid arthritis. $J$ Rheumatol 2005;32:1219-23.

12. Sirdah MM, Abu Ghali AS, Al Laham NA. The reliability of the National Cholesterol Education Program's Adult Treatment Panel III (NCEP/ATP III) and the International Diabetes Federation (IDF) definitions in diagnosing metabolic syndrome (MetS) among Gaza Strip Palestinians. Diabetes Metab Syndr 2012;6:4-8.

13. Gunes F, Akbal E, Asik M, Sen H, Binnetoglu E, Kizilgun $\mathrm{M}$, et al. Number of metabolic syndrome risk parameters associated with TAFIa/ai antigen levels. Blood Coagul Fibrinolysis 2013;24:844-7.

14. del Rincón ID, Williams K, Stern MP, Freeman GL, Escalante A. High incidence of cardiovascular events in a rheumatoid arthritis cohort not explained by traditional cardiac risk factors. Arthritis Rheum 2001;44:2737-45.

15. Simons PC, Algra A, Bots ML, Grobbee DE, van der Graaf Y. Common carotid intima-media thickness and arterial stiffness: indicators of cardiovascular risk in high-risk patients. The SMART Study (Second 
Manifestations of ARTerial disease). Circulation 1999;100:951-7.

16. Gonzalez-Juanatey C, Llorca J, Testa A, Revuelta J, Garcia-Porrua C, Gonzalez-Gay MA. Increased prevalence of severe subclinical atherosclerotic findings in long-term treated rheumatoid arthritis patients without clinically evident atherosclerotic disease. Medicine (Baltimore) 2003;82:407-13.

17. Turkyilmaz AK, Devrimsel G, Kirbas A, Cicek Y, Karkucak M, Capkin E, et al. Relationship between pulse wave velocity and serum YKL-40 level in patients with early rheumatoid arthritis. Rheumatol Int 2013;33:2751-6.

18. Del Rincón I, O'Leary DH, Freeman GL, Escalante A. Acceleration of atherosclerosis during the course of rheumatoid arthritis. Atherosclerosis 2007;195:354-60.

19. Park YB, Ahn CW, Choi HK, Lee $\mathrm{SH}$, In BH, Lee $\mathrm{HC}$, et al. Atherosclerosis in rheumatoid arthritis: morphologic evidence obtained by carotid ultrasound. Arthritis Rheum 2002;46:1714-9.

20. Salonen R, Tervahauta M, Salonen JT, Pekkanen $J$, Nissinen A, Karvonen MJ. Ultrasonographic manifestations of common carotid atherosclerosis in elderly eastern Finnish men. Prevalence and associations with cardiovascular diseases and risk factors. Arterioscler Thromb 1994;14:1631-40.

21. Solomon DH, Kremer J, Curtis JR, Hochberg MC, Reed G, Tsao P, et al. Explaining the cardiovascular risk associated with rheumatoid arthritis: traditional risk factors versus markers of rheumatoid arthritis severity. Ann Rheum Dis 2010;69:1920-5.

22. Galarza-Delgado DA, Esquivel-Valerio JA, GarzaElizondo MA, Góngora-Rivera F, Muñoz-De Hoyos JL, Serna-Peña G.Carotid atherosclerosis in patients with rheumatoid arthritis and rheumatoid nodules. Reumatol Clin 2013;9:136-41. 